\title{
The Clinical and Economic Benefits of Co-Testing Versus Primary HPV Testing for Cervical Cancer Screening: A Modeling Analysis
}

\author{
Juan C. Felix, MD,' Michael J. Lacey, MS, Jeffrey D. Miller, MS, Gregory M. Lenhart, MS, \\ Mark Spitzer, MD, and Rucha Kulkarni, MHS ${ }^{2}$
}

\begin{abstract}
Background: Consensus United States cervical cancer screening guidelines recommend use of combination Pap plus human papillomavirus (HPV) testing for women aged 30 to 65 years. An HPV test was approved by the Food and Drug Administration in 2014 for primary cervical cancer screening in women age 25 years and older. Here, we present the results of clinical-economic comparisons of Pap plus HPV mRNA testing including genotyping for HPV 16/18 (co-testing) versus DNA-based primary HPV testing with HPV 16/18 genotyping and reflex cytology (HPV primary) for cervical cancer screening.

Methods: A health state transition (Markov) model with 1-year cycling was developed using epidemiologic, clinical, and economic data from healthcare databases and published literature. A hypothetical cohort of one million women receiving triennial cervical cancer screening was simulated from ages 30 to 70 years. Screening strategies compared HPV primary to co-testing. Outcomes included total and incremental differences in costs, invasive cervical cancer (ICC) cases, ICC deaths, number of colposcopies, and quality-adjusted life years for cost-effectiveness calculations. Comprehensive sensitivity analyses were performed.

Results: In a simulation cohort of one million 30-year-old women modeled up to age 70 years, the model predicted that screening with HPV primary testing instead of co-testing could lead to as many as 2,141 more ICC cases and 2,041 more ICC deaths. In the simulation, co-testing demonstrated a greater number of lifetime quality-adjusted life years $(22,334)$ and yielded $\$ 39.0$ million in savings compared with HPV primary, thereby conferring greater effectiveness at lower cost.

Conclusions: Model results demonstrate that co-testing has the potential to provide improved clinical and economic outcomes when compared with HPV primary. While actual cost and outcome data are evaluated, these findings are relevant to U.S. healthcare payers and women's health policy advocates seeking cost-effective cervical cancer screening technologies.
\end{abstract}

\section{Introduction}

$\mathbf{O}$ VER THE PAST SEVERAL DECADES, cervical cancer screening in the United States has reduced mortality rates of cervical cancer from the leading cause of cancer death in women to ranking 14th. Increased adoption of the Pap test as a screening tool was largely responsible for the decrease through improved detection and treatment of cervical intraepithelial neoplasia (CIN). ${ }^{1}$ Yet even with the success of cervical cancer screening, it is estimated that this year in the United States there will be 12,900 new cases of cervical cancer and 4,100 women will die of this disease. ${ }^{2}$ Technological advances such as vaccination and testing for human papillomavirus (HPV) have the potential to decrease cervical cancer rates even further. ${ }^{3-6}$ Because of its greater sensitivity, combination Pap plus HPV testing is the preferred screening strategy for women 30 to 65 years of age compared with HPV testing or Pap testing alone, while Pap testing is

\footnotetext{
${ }^{1}$ Keck School of Medicine, University of Southern California, Los Angeles, California.

${ }^{2}$ Truven Health Analytics, Cambridge, Massachusetts.

${ }^{3}$ Hofstra North Shore-LIJ School of Medicine, Hempstead, New York.

(C) Juan C. Felix, et al. 2016; Published by Mary Ann Liebert, Inc. This Open Access article is distributed under the terms of the Creative Commons Attribution Noncommercial License (http://creativecommons.org/licenses/by-nc/4.0/) which permits any noncommercial use, distribution, and reproduction in any medium, provided the original author(s) and the source are credited.
} 
recommended for women aged 21 to 30 due to the lower specificity of HPV testing in this population. ${ }^{7}$ Controversies regarding appropriate cervical screening intervals and the recent United States Food and Drug Administration approval of an HPV test as a primary cervical cancer screen have raised questions among patients, clinicians, and payers regarding the clinical and cost effectiveness of various cervical cancer screening strategies. 8,9

Data from several European trials has shown effectiveness for HPV primary screening, with some studies concluding that HPV primary testing can support screening intervals as long as 6 years. ${ }^{10,11}$ Additionally, a cost-effectiveness study performed using the results of the ARTISTIC trial determined that HPV testing would be cost effective as a primary screen. ${ }^{12}$ However, these studies have generally compared HPV primary screening with Pap testing rather than Pap plus HPV testing and have been performed in populations with single-payer healthcare and differing screening utilization compared with the United States. ${ }^{13,14}$ Long-term, largepopulation studies investigating the cost effectiveness and clinical impact of HPV primary screening are necessary to determine the effectiveness of HPV primary screening in the United States compared with the current clinical practice of Pap plus HPV testing.

At least one attempt has been made to use modeling data to estimate the cost effectiveness of different cervical screening strategies in the United States. ${ }^{15}$ However, several factors that may affect the cost effectiveness of HPV primary screening have not been investigated. First, the cost effectiveness of HPV testing in women aged 25 to 29 has not been assessed. Because the Food and Drug Administration approved an HPV primary test for women $\geq 25$ years of age, ${ }^{9}$ a model including various screening strategies at different ages would provide practical insights into the cost effectiveness of HPV primary testing in varying age populations where it may be used. Second, while the previous analysis compared Pap plus HPV without genotyping to HPV primary with genotyping, the impact of HPV genotyping on cost effectiveness has not previously been incorporated into economic modeling when comparing Pap plus HPV testing with HPV primary screening. ${ }^{15}$ Reflex genotyping for HPV 16/18 following Pap plus HPV testing is recommended by the major guidelines (when the result is a negative Pap and a positive HPV test), ${ }^{7}$ so comparing Pap plus HPV screening paradigms that include genotyping with an HPV primary screening paradigm that includes genotyping is necessary for a like comparison. Third, while the previously published model analyzed Pap plus HPV testing using a DNA-based assay, the majority of US diagnostic labs use mRNA-based HPV testing performed with Pap. ${ }^{16}$

Although guidelines currently recommend a 5-year screening interval for Pap plus HPV, this continues to be the subject of ongoing debate in the scientific community as clinical data ${ }^{5}$ and modeling analyses from the U.S. Preventative Services Task Force (USPSTF) suggest that screening intervals longer than 3 years may result in substantial increases in cervical cancer morbidity and mortality. ${ }^{8,17}$ The USPSTF model developed by Kulasingam et al. found that co-testing intervals of 3 versus 5 years would result in 4.73 versus 7.44 cases of cervical cancer and 0.74 versus 1.35 deaths per 1,000 women, increasing invasive cervical cancer (ICC) cases by $57 \%$ and cervical cancer deaths by $82 \% .{ }^{17}$ Kinney et al. ${ }^{8}$ calculated that this increase predicts that adopting 5-year screening intervals will result in an additional 1 in 369 screening-compliant women contracting ICC and 1 in 1,639 screening-compliant women dying of cervical cancer. Additionally, surveys of practice patterns suggest that the majority of clinicians continue to screen with Pap plus HPV more often than every 5 years, ${ }^{18-20}$ versus the 2012 guidelines, which recommended a 5-year interval. ${ }^{7}$ Because of these data and published guidance for HPV primary recommending that "re-screening after a negative primary highrisk HPV screen should occur no sooner than every 3 years,",21 we believe that a comparison of Pap plus HPV (i.e., "cotesting") and HPV primary with equivalent intervals of 3 years is most clinically relevant.

We attempted to address the limitations of previous work with the objective of performing clinical and economic comparisons between cervical cancer screening with Pap plus HPV mRNA testing with genotyping for HPV 16/18 (cotesting) versus primary HPV DNA testing with genotyping for HPV 16/18 and reflex cytology (HPV primary). We present the results of an analysis using a hypothetical cohort of one million 30-year-old women modeled up to age 70 in which co-testing and HPV primary were assessed by comparing the number of cases and deaths from invasive cervical cancer (ICC), expected and predicted quality-adjusted life years (QALYs), and estimated costs associated with each screening strategy. We also present the results of sensitivity analyses investigating the effects of test performance characteristics, mortality rates, and a comparison of HPV primary testing with Pap testing in women 25 to 29 years of age.

\section{Methods}

\section{Economic model overview}

In this study, we perform clinical-economic comparisons of two cervical cancer screening strategies: Pap plus HPV mRNA testing with genotyping (co-testing) and primary HPV DNA testing with genotyping and reflex cytology (HPV primary). The model estimates the impact within a cohort of one million 30-year-old women receiving high-risk HPV screening. Outcomes and costs were simulated from ages 30 to 70 years. Differences in outcomes between the two scenarios were accumulated annually across each cohort's life span. Outcomes included total and incremental differences in costs, ICC cases, ICC deaths, and QALYs for costeffectiveness calculations. Data for this study was derived from published literature and analysis of insurance claims records.

\section{Study population}

To conduct the evaluation, we constructed a hypothetical cohort of one million women with cervical cancer risk representative of today's population who would be eligible to begin cervical cancer screening at age 30 years per consensus guidelines. ${ }^{7}$ The cohort was assumed to experience the same natural history of disease, screening, diagnosis and treatment in a U.S. healthcare setting and followed from age 30 years to age 70 years. A secondary analysis of women aged 25 to 29 years was also performed.

Development of ICC has a long latency period and nearly all cases are associated with persistent high-risk HPV subtypes. $^{22}$ Key clinical attributes include high rates of natural 
clearance of HPV infection and age-dependent persistence among these strains. Disease progression follows a defined series of stages, from initial HPV infection through progressive stages of cervical lesion development prior to the appearance of cervical cancer. ${ }^{23}$

\section{Screening strategies}

We modeled cervical cancer screening strategies using concurrent ThinPrep liquid-based cytology and high-risk HPV mRNA testing (ThinPrep ${ }^{\circledR}$ Pap Test and Aptima ${ }^{\circledR}$ HPV Assay, Hologic, Inc.) with HPV16 18/45 genotyping (cotesting) compared with primary high-risk HPV DNA testing with HPV 16/18 genotyping (cobas ${ }^{\circledR}$ HPV test, Roche Diagnostics US) with reflex for women testing HPV positive but HPV 16/18 negative to ThinPrep liquid-based cytology (HPV primary). Women were assumed to be screened once every 3 years for either screening strategy. ${ }^{7,8,19}$ In the model, women testing positive entered a 1-year retesting cycle until a negative screen was observed; colposcopies were performed on women testing positive for (a) HPV 16/18; (b) other highrisk HPV and cytology $\geq$ "atypical squamous cells of undetermined significance" (ASCUS); (c) cytology $\geq$ "low grade squamous intraepithelial lesion" irrespective of the HPV results; or (d) other high-risk HPV for two consecutive screening tests. Colposcopies were assumed to be $100 \%$ sensitive and specific for CIN2/3. Compliance with periodic screening was held constant at $100 \%$ for all testing strategies for the sake of equalizing the analytical comparisons and eliminating bias against tests with lower sensitivity from a factor exogenous to the focus of our analyses. In addition, we chose not to incorporate adherence into the modeling analyses because one-half of the 12,000 women diagnosed with cervical cancer in the United States in 2012 had not been screened within 5 years, ${ }^{24}$ and screening compliance varies widely and is dependent upon factors such as age, education, and socioeconomic status. ${ }^{25}$ With consideration of this important evidence, we felt that incorporating treatment adherence had the potential for generating misleading results rather than adding to the clinical relevance of our approach.

\section{Model structure}

A health state transition (Markov) model with 1-year cycling was developed in TreeAge Pro 2015 (TreeAge Software, Inc.) using epidemiologic, clinical, and economic data from healthcare databases and published literature. Numerous researchers have developed population-level natural history models of the development and progression from initial HPV status through development of ICC. ${ }^{23,26,27}$ The conceptual foundation and structure of these models are similar and have served as the basis for health policy and guidelines development over the past 15 to 20 years. $^{23}$ Probabilities used to model natural history of viral clearance, disease progression, and regression were taken from a large federally funded epidemiology modeling program housed at the USPSTF, ${ }^{23}$ except where noted.

In this health-state transition model, cohort women were assumed to be in one of seven mutually exclusive health states: HPV HR (-), HPV HR (+), CIN1, CIN2, CIN3, ICC, and dead. Within the dead state, patients could either die from ICC or all other causes (Supplementary Fig. S1; Supplementary Data are available online at www.liebertpub.com/ jwh). At the end of each annual cycle, women could either remain in the current state, transition to a more severe disease state or death, or transition to a less severe health state. Agespecific transition probabilities of high-risk HPV infection, CIN clearance, progression, and regression are detailed in Table 1 .

\section{Model inputs}

Test performance was defined as the sensitivity and specificity of the overall screening strategy and is detailed in Table 2. Base case sensitivity and specificity estimates were defined based on previously reported values for detecting CIN3. ${ }^{28}$ Because these values were derived from a study that evaluated co-testing utilizing cytology review without automated imaging and HPV testing utilizing a DNA-based assay, the following adjustments were made: co-testing sensitivity was increased from $76.2 \%$ to $82.0 \%$ to account for the increased sensitivity of cytology with automated imaging; ${ }^{29}$ and co-testing specificity was increased from $81.5 \%$ to $85.3 \%$ to account for the increased specificity of mRNAbased HPV testing. ${ }^{30-33}$

Costs were modeled from the perspective of a U.S. healthcare payer and are reported in 2014 U.S. dollars. Our cost accounting approach was comprehensive and included all costs associated with diagnostic tests and procedures (including colposcopy and biopsy), additional and repeat testing, medical office visits, cancer treatment costs, and endof-life care. Costs for diagnostic tests and procedures were derived based on the number of diagnostic tests performed and unit cost data from current payment levels using the Truven Health Analytics MarketScan ${ }^{\circledR}$ Research Databases (Truven Health Analytics). For example, the cost for HPV primary screening included the sum of the test cost and the cost of additional reflex testing based on disease prevalence and test performance characteristics. Treatment costs for CIN2 or CIN3 were taken from a recent study on the costs of care for these patients, ${ }^{34}$ and treatment of CIN2/3 was assumed to be $100 \%$ successful. CIN1 was assumed to not be treated and therefore did not incur added costs if discovered. Costs for treating ICC were split into three components to account for high initial costs of care and lower costs in subsequent years for surviving patients. ${ }^{35}$ For terminal ICC patients, an additional cost accounting for end-of-life care was added to either the initial or subsequent year depending on the age of death. ${ }^{35}$

\section{Model outcomes}

The primary outcome measure was total direct medical costs per woman screened. Direct costs as defined above (screening, diagnostic follow-up, and treatment), were summed during each year and summed over the course of 40 years from 30 to 70 years of age. Additional outcomes included the cumulative number of ICC cases, ICC-related deaths, number of colposcopies, overall cost of screening and diagnosis, and overall cost of treatment.

We also examined the cost effectiveness of each screening strategy using the cost per QALY. Cost effectiveness is represented as incremental cost-effectiveness ratio (ICER). This ratio represents the efficiency of a screening strategy compared with the next best alternative. We defined an ICER threshold of US\$50,000 per QALY as good value for a U.S. 


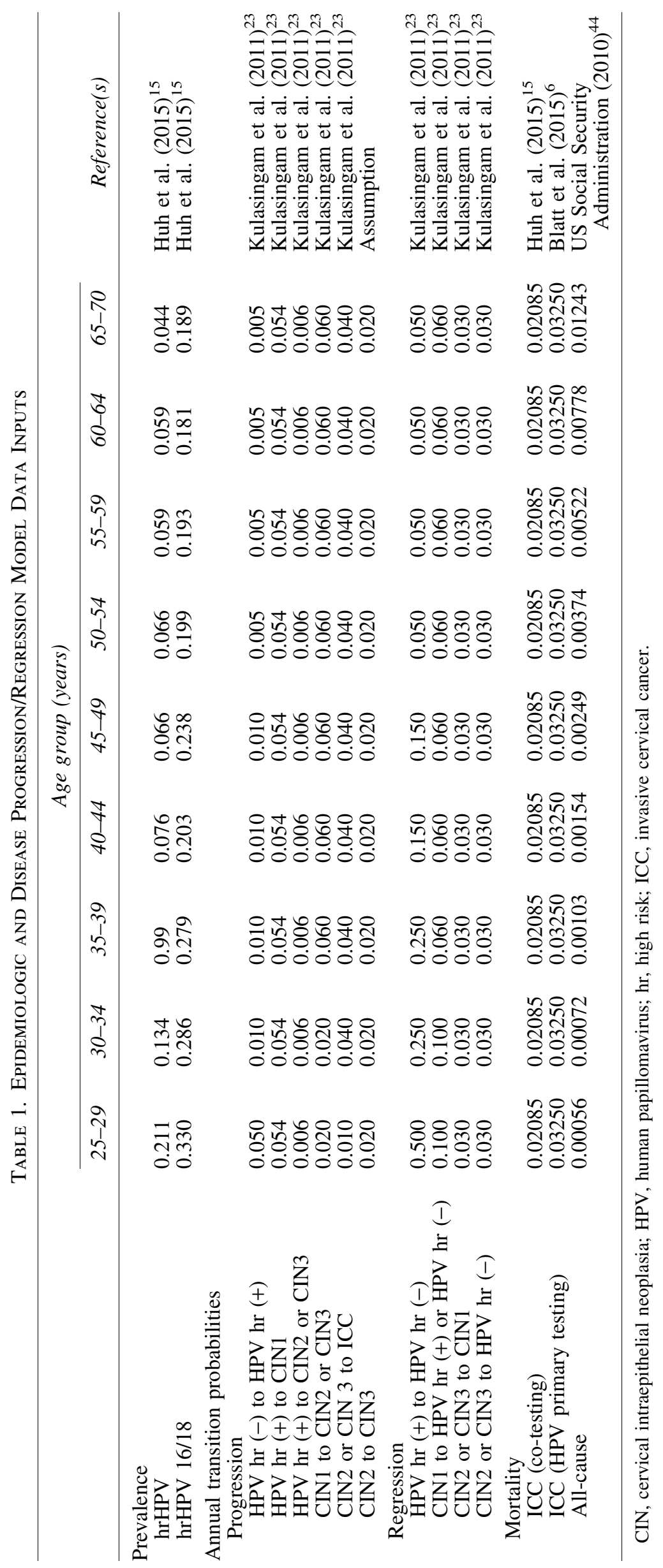


Table 2. Costs, Sensitivity/Specificity, and Health Utilities Model Inputs

\begin{tabular}{|c|c|c|}
\hline Variable & Base case & Reference(s) \\
\hline $\begin{array}{l}\text { Annual discount rate } \\
\text { Costs and QALYs }\end{array}$ & $3.0 \%$ & Assumption \\
\hline $\begin{array}{l}\text { Unit costs (2014 USD)* } \\
\text { Treatment of CIN2 or CIN3 } \\
\text { Colposcopy plus biopsy } \\
\text { Cytology } \\
\text { HPV hr genotyping } \\
\text { HPV hr pooled test } \\
\text { Office visit (routine/repeat screening) } \\
\text { Treatment of ICC (initial) } \\
\text { Treatment of ICC (continuing) } \\
\text { Treatment of ICC (terminal) }\end{array}$ & $\begin{array}{c}\$ 43.58 \\
\$ 43.58 \\
\$ 74.55 \\
\$ 55,684 \\
\$ 1,597 \\
\$ 110,033\end{array}$ & $\begin{array}{l}\text { Vijayaraghavan et al. }(2010)^{34} \\
\text { Huh et al. }(2015) \\
\text { 50/50 blended of image/nonimaged slides; } \\
\text { CPT codes } 88142,88175 \\
\text { CPT code } 87624 \\
\text { CPT code } 87624 \\
\text { Huh et al. }(2015),{ }^{15} \text { CPT code } 88305 \\
\text { Mariotto et al. }(2011)^{35} \\
\text { Mariotto et al. }(2011)^{35} \\
\text { Mariotto et al. }(2011)^{35}\end{array}$ \\
\hline $\begin{array}{l}\text { Sensitivity }{ }^{\dagger} \\
\text { Co-testing }\end{array}$ & 0.680 & 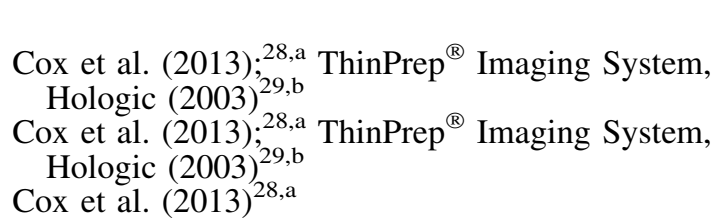 \\
\hline $\begin{array}{l}\text { Cytology } \\
\text { HPV primary }\end{array}$ & $\begin{array}{l}0.876 \\
0.852\end{array}$ & $\begin{array}{l}\text { Cox et al. }(2013) ;^{28, a} \text { Ting et al. }(2015) ;^{30, c} \\
\quad \text { Monsonego et al. }(2011) ;^{31, d} \text { Iftner et al. } \\
\quad(2015) ; ;^{13, \mathrm{e}} \text { APTIMA }{ }^{\circledR} \text { SSED, Hologic }(2013)^{33, \mathrm{f}} \\
\text { Cox et al. }(2013)^{28, \mathrm{a}} \\
\text { Cox et al. }(2013)^{28, \mathrm{a}}\end{array}$ \\
\hline $\begin{array}{l}\text { Five-year survival rate }{ }^{\dagger} \\
\text { Co-testing } \\
\text { HPV primary } \\
\text { Cytology }\end{array}$ & $\begin{array}{l}90 \% \\
85 \% \\
85 \%\end{array}$ & $\begin{array}{l}\text { Howlander et al. (2012) })^{45} \text {; Blatt et al. }(2015)^{6, g} \\
\text { Howlander et al. }(2012)^{45} \text {; Blatt et al. }(2015)^{6, g} \\
\text { Howlander et al. }(2012)^{45} \text {; Blatt et al. }(2015)^{6, g}\end{array}$ \\
\hline $\begin{array}{l}\text { Health utilities } \\
\text { Well } \\
\text { CIN1 } \\
\text { CIN2 } \\
\text { CIN3 } \\
\text { ICC }\end{array}$ & $\begin{array}{l}1.00 \\
0.97 \\
0.97 \\
0.97 \\
0.71\end{array}$ & $\begin{array}{l}\text { Huh et al. }(2015)^{15} \\
\text { Huh et al. }(2015)^{15} \\
\text { Huh et al. }(2015)^{15} \\
\text { Huh et al. }(2015)^{15} \\
\text { Huh et al. }(2015)^{15}\end{array}$ \\
\hline
\end{tabular}

*Costs were adjusted to 2014 U.S. dollars (USD) using the medical care component of the Consumer Price Index.

${ }^{\dagger}$ Estimated variable values.

${ }^{a}$ Study included ThinPrep ${ }^{\circledR}$ Pap Test without the ThinPrep ${ }^{\circledR}$ Imaging System, HPV Linear Array ${ }^{\circledR}$ HPV Genotyping Test, Amplicor ${ }^{\circledR}$ HPV Test, and cobas ${ }^{\circledR}$ HPV Test.

${ }^{b}$ Study included ThinPrep ${ }^{\circledR}$ Pap Test slides assessed with either the ThinPrep ${ }^{\circledR}$ Imaging System or manual review.

${ }^{\mathrm{C}}$ Study included modeled results for ThinPrep ${ }^{\circledR}$ Pap Test and Aptima ${ }^{\circledR}$ HPV and Hybrid Capture ${ }^{\circledR} 2$ assays.

${ }^{\mathrm{d}}$ Study included ThinPrep ${ }^{\circledR}$ Pap Test and Aptima ${ }^{\circledR}$ HPV and Hybrid Capture ${ }^{\circledR} 2$ assays.

${ }^{\mathrm{e}}$ Study included ThinPrep ${ }^{\circledR}$ Pap Test and Aptima ${ }^{\circledR}$ HPV, Hybrid Capture ${ }^{\circledR}$ 2, and INNO-LiPA ${ }^{\circledR}$ HPV Genotyping Extra assays.

${ }^{\mathrm{f}}$ Study included ThinPrep ${ }^{\circledR}$ Pap Test and Aptima ${ }^{\circledR}$ HPV and 16 18/45 GT assays.

${ }^{\mathrm{g}}$ Study included ThinPrep Pap Test ${ }^{\circledR}$, SurePath ${ }^{\mathrm{TM}}$ Pap Test, and Hybrid Capture ${ }^{\circledR} 2$ assay.

CPT, common procedural terminology; QALY, quality-adjusted life-year.

payer, which has been described elsewhere. ${ }^{36}$ QALY weights associated with each health state in our model are summarized in Table 2 .

\section{Nationwide projection}

For policy makers, we also projected the potential economic and clinical impact of each screening strategy on the total U.S. population. For each outcome, total costs, ICC cases, and ICC deaths, we first ran the model for individual cohorts in 5-year increments (e.g., starting at age 30 for 40 years, age 35 for 35 years, age 40 for 30 years, etc.) to reflect the impact that could be expected if a scenario was implemented immediately across a cross-section of the U.S. population. For each outcome, the model generates the marginal impact for the age group. Total costs or ICC cases and ICC deaths equal the marginal impact estimated in each age-stratified cohort times the female U.S. population in each age category. Net impact was estimated based on the net difference summed across the population.

\section{Sensitivity analyses}

We conducted one-way deterministic sensitivity analyses on selected parameters, including test performance characteristics, prevalence rates, mortality rates, and costs. In addition to comparing co-testing to HPV primary in women $\geq 30$ years of age, we also explored the impact of HPV primary 
Table 3. Outcomes, Costs, and Cost Effectiveness for the Two Cervical Cancer Screening Strategies

\begin{tabular}{lccc}
\hline & \multicolumn{2}{c}{ Ages 30-70 years } \\
\cline { 2 - 4 } Outcome & Co-testing & HPV primary & Net difference \\
\hline ICC cases per 10,000 & 57.61 & 79.02 & -21.41 \\
ICC deaths per 10,000 & 23.06 & 43.47 & -20.41 \\
Lifetime QALYs & 23.0084 & 22.9861 & 0.0223 \\
Screening costs (USD) & $\$ 1,319$ & $\$ 1,129$ & $\$ 189$ \\
Treatment costs (USD) & $\$ 1,007$ & $\$ 1,236$ & $-\$ 228$ \\
Total costs (USD) & $\$ 2,326$ & $\$ 2,365$ & $-\$ 39$ \\
ICER $^{\mathrm{a}}$ (USD) & & & $-\$ 1,725$ (co-testing dominant)
\end{tabular}

${ }^{\mathrm{a}} \mathrm{Net}$ difference $=$ Co-testing - HPV primary.

${ }^{\mathrm{b}} \mathrm{QALYs}$ reported as per woman screened.

${ }^{\mathrm{c}}$ Incremental cost-effectiveness ratio $(\mathrm{ICER})=\Delta$ Cost $/ \triangle \mathrm{QALY}$.

versus cytology with ASCUS reflex to high-risk HPV among women aged 25 to 29 years who initiate screening at age 25. Estimated rates of disease progression and regression and test-performance characteristics vary between the USPSTF and other recently published models (Supplementary Table S1). ${ }^{15,23}$ We compared the outcomes among this patient population to determine the potential impact of initiating frontline screening with HPV at age 25.

\section{Results}

\section{Model results}

The results of the model analyses comparing co-testing with HPV primary are shown in Table 3 . The patient population for the model analysis is composed of a hypothetical cohort of one million women aged 30 years undergoing alternative cervical screening strategies over 40 years. Lifetime cumulative costs of screening and management per patient equaled $\$ 2,326$ for co-testing and $\$ 2,365$ for HPV primary. For co-testing, screening and diagnosis costs equal $\$ 1,319$ and $\$ 1,007$ respectively, while for HPV primary, they are $\$ 1,129$ and $\$ 1,236$ respectively. The model calculated lower overall total costs associated with co-testing compared with HPV primary, representing an overall savings of $\$ 39$ over the course of a woman's lifetime.

Co-testing resulted in a slight decrease in lifetime colposcopies per woman compared with HPV primary (2.227 vs. 2.232 respectively), representing 4,557 fewer colposcopies in the one-million-woman cohort. In addition, co-testing resulted in more true positive colposcopies per woman compared with HPV primary ( 0.127 vs. 0.122 respectively) and fewer false positive colposcopies (2.101 vs. 2.111). These numbers represent 5,206 more true positive colposcopies and 9,763 fewer false positive colposcopies across the entire cohort.

HPV primary testing was calculated to result in as many as 21 additional ICC cases per 10,000 women screened and 20 additional ICC deaths per 10,000 compared with co-testing. The model also calculated a greater number of QALYs per women screened for co-testing at 23.01 compared with HPV primary at 22.99 . These results demonstrate a greater effectiveness and lower cost of co-testing that dominates the HPV primary screening strategy.

With the results translated to the one-million-woman cohort, the model predicted that as many as 2,141 ICC cases and 2,041 ICC deaths could be prevented with co-testing vs HPV primary screening. Co-testing demonstrated a greater number of lifetime QALYs $(22,334)$ and yielded $\$ 39.0$ million in savings compared with HPV primary.

\section{Nationwide projection}

The model results were used to calculate a projection to demonstrate the impact at the U.S. national level (Table 4). Using a cross section of the total U.S. female population of 78.9 million women between 30 and 70 years of age, ${ }^{37}$ the

Table 4. Net Cost Savings Projected to the National Level

\begin{tabular}{|c|c|c|c|c|c|c|c|c|}
\hline \multirow{2}{*}{$\begin{array}{l}\text { Age } \\
\text { (years) }\end{array}$} & \multirow{2}{*}{$\begin{array}{c}\text { Co- } \\
\text { testing }\end{array}$} & \multirow{2}{*}{$\begin{array}{c}H P V \\
\text { primary }\end{array}$} & \multirow{2}{*}{$\begin{array}{c}\text { Net } \\
\text { difference }^{\mathrm{a}}\end{array}$} & \multirow{2}{*}{$\begin{array}{c}\text { Female U.S. } \\
\text { population }\end{array}$} & \multirow{2}{*}{$\begin{array}{l}\text { Population } \\
\text { distribution }\end{array}$} & \multicolumn{2}{|c|}{ Total costs } & \multirow{2}{*}{$\begin{array}{l}\text { Lifetime cost } \\
\text { savings }{ }^{b}\end{array}$} \\
\hline & & & & & & Co-testing & $H P V$ primary & \\
\hline $30-34$ & $\$ 2,326$ & $\$ 2,365$ & $-\$ 39$ & $9,965,599$ & $13 \%$ & $\$ 23,179,983,274$ & $\$ 23,568,641,635$ & $-\$ 388,658,361$ \\
\hline & $\$ 2,298$ & $\$ 2,370$ & $-\$ 72$ & & & $\$ 23,296,250,760$ & & \\
\hline $40-44$ & $\$ 2,130$ & $\$ 2,207$ & $-\$ 77$ & $10,496,987$ & $13 \%$ & $\$ 22,358,582,310$ & $\$ 23,166,850,309$ & $-\$ 808,267,999$ \\
\hline & $\$ 1,917$ & $\$ 1,991$ & $-\$ 74$ & $11,499,506$ & $15 \%$ & $\$ 22,044,553,002$ & $\$ 22,895,516,446$ & $-\$ 850,963,444$ \\
\hline $50-54$ & $\$ 1,688$ & $\$ 1,755$ & $-\$ 67$ & $11,364,851$ & $14 \%$ & $\$ 19,183,868,488$ & $\$ 19,945,313,505$ & $-\$ 761,445,017$ \\
\hline $55-59$ & $\$ 1,383$ & $\$ 1,438$ & $-\$ 55$ & $10,141,157$ & $13 \%$ & $\$ 14,025,220,131$ & $\$ 14,582,983,766$ & $-\$ 557,763,635$ \\
\hline $60-64$ & $\$ 1,067$ & $\$ 1,102$ & $-\$ 35$ & $8,740,424$ & $11 \%$ & $\$ 9,326,032,408$ & $\$ 9,631,947,248$ & $-\$ 305,914,840$ \\
\hline $65-69$ & $\$ 666$ & $\$ 672$ & $-\$ 6$ & $6,582,716$ & $8 \%$ & $\$ 4,384,088,856$ & $\$ 4,423,585,152$ & $-\$ 39,496,296$ \\
\hline Total & & & & $78,928,860$ & & & & $-\$ 4,442,418,232$ \\
\hline
\end{tabular}

${ }^{\mathrm{a} N e t}$ difference $=$ Co-testing - HPV primary.

${ }^{\mathrm{b}}$ Lifetime cost savings $=$ Total co-testing costs - Total HPV primary costs. 
Table 5. Sensitivity Analysis Values

\begin{tabular}{|c|c|c|c|}
\hline Parameter & Base case value & Lower range & Upper range \\
\hline Sensitivity of co-testing & $82 \%$ & $76.2 \%$ & $85 \%$ \\
\hline Specificity of co-testing & $85.3 \%$ & $81.5 \%$ & $88.7 \%$ \\
\hline Sensitivity of HPV primary & $72 \%$ & $67.8 \%$ & $76.2 \%$ \\
\hline Specificity of HPV primary & $85.2 \%$ & $81.5 \%$ & $88.7 \%$ \\
\hline Five-year survival for ICC (HPV primary)* & $85 \%$ & - & $90 \%$ \\
\hline Cost of colposcopy biopsy & $\$ 304.78$ & $\$ 274.30$ & $\$ 335.26$ \\
\hline Cost of HPV genotyping & $\$ 43.58$ & $\$ 39.22$ & $\$ 47.94$ \\
\hline Cost of HPV pooled testing & $\$ 43.58$ & $\$ 39.22$ & $\$ 47.94$ \\
\hline Cost of ICC incremental & $\$ 1,597$ & $\$ 1,437.30$ & $\$ 1,756.70$ \\
\hline Cost of ICC initial & $\$ 55,684$ & $\$ 50,115.60$ & $\$ 61,252.40$ \\
\hline Cost of ICC terminal & $\$ 110,033$ & $\$ 99,029.70$ & $\$ 121,036.30$ \\
\hline Cost of office visit & $\$ 74.55$ & $\$ 67.10$ & $\$ 82.01$ \\
\hline Cost of CIN2 \& CIN3 & $\$ 1,345$ & $\$ 1,210.50$ & $\$ 1,479.50$ \\
\hline
\end{tabular}

$*$ The base case value of 5-year ICC survival is $90 \%$ for co-testing and $85 \%$ for HPV primary; the sensitivity analysis tested the impact of varying 5-year ICC survival for HPV primary to $90 \%$.

model predicts approximately 150,000 additional ICC cases and more than 100,000 additional ICC deaths using an HPV primary approach compared with co-testing. This represents an overall additional lifetime cost of approximately $\$ 4.4$ billion for HPV primary.

\section{One-way sensitivity analysis}

A one-way sensitivity analysis was performed on the model to test the influence of several key parameters. The primary factors tested were test performance for co-testing and HPV primary screening, costs of the tests and treatments, and 5-year survival for invasive cervical cancer. The ranges of values used for each of the factors in the sensitivity analysis can be found in Table 5 .

Tornado diagrams were created for each of the four major outcomes of interest: cumulative total costs; QALYs; ICC cases per 10,000; and ICC deaths per 10,000 women. All four analyses show that both clinical and cost outcomes are highly sensitive to the performance of the screening tests. Figure 1A shows the results of the one-way sensitivity analysis for the net difference of the cumulative total costs. The difference in cumulative total costs between co-testing and HPV Primary screening ranged from $-\$ 179$ per woman (i.e., cost savings conferred by co-testing) to $\$ 111$ per woman (i.e., additional cost conferred by co-testing). Total costs were most sensitive to the specificity of co-testing, followed by specificity of HPV primary, sensitivity of HPV primary, and then sensitivity of co-testing. When the specificity of co-testing approached the high value of $88.7 \%$, co-testing saved $\$ 166$ per woman when compared with the base case savings of $\$ 39$. When the specificity of co-testing was set to $81.5 \%$, the specificity of DNA-based co-testing reported by Cox et al., ${ }^{28}$ co-testing resulted in an incremental cost of $\$ 111$ per woman. In the other three analyses, the most influential parameter was the sensitivity of HPV primary followed by the sensitivity of co-testing.

Figure 1B-D shows the results of the one-way sensitivity analysis varying the test performance characteristics of cotesting and HPV primary for the clinical outcomes of QALYs, ICC cases, and ICC deaths. In the simulation, sensitivity analysis demonstrated that co-testing provided clinically superior outcomes (greater QALYs, fewer ICC cases, fewer
ICC deaths) compared with HPV primary across the entire range of inputs.

\section{Sensitivity analysis for women 25-29 years old}

A second sensitivity analysis was performed to determine the impact of transition probabilities cited in Huh et al. ${ }^{15}$ and Kulasingam et al. ${ }^{23}$ on women aged 25 to 29 years. Huh et al. generally cites a higher rate of progression to the various health states as well as a higher rate of regression when compared with the USPSTF data used by Kulasingam et al. (Supplementary Table S1). ${ }^{15,23}$

Overall, both sets of transition probabilities demonstrate that cytology with ASCUS reflex has greater cost savings in our model; however, cytology has a slightly higher number of ICC cases but not ICC mortality when compared with HPV primary (Supplementary Table S2). Use of USPSTF data progression tables resulted in a greater number of net ICC cases per 10,000 compared with using the Huh progression tables (0.51 vs. 0.26). Both progression tables found a similar number of net ICC deaths per 10,000 (0.03 vs. 0.02). Using the Huh data, HPV primary generated an ICER of $\$ 611,818$ compared with an ICER of \$426,667 with USPSTF data, indicating that HPV primary is not cost effective compared with cytology in women screened between the ages of 25 and 29 years using either USPSTF or Huh data as inputs (Supplementary Table S2).

\section{Sensitivity analysis for ICC mortality calibrated to Surveillance, Epidemiology, and End Results Program data}

A third sensitivity analysis was performed to test the impact of calibrating ICC mortality rates (Table 1), which were held constant in the model for all simulation years in the base-case analysis, against multiyear survival curves derived from Surveillance, Epidemiology, and End Results (SEER) Program cervical cancer data to reflect the tapering effect observed in AJCC stage 1 survival after 5 years following diagnosis. ${ }^{38}$ To replicate this effect in the model data, all ICC mortality rates were reduced by $50 \%$ after the fifth year of simulation post diagnosis (i.e., the ICC mortality rates in Table 1 were halved for women with ICC diagnosis who survived to year 6 and beyond). The impact of making this change on modeling 


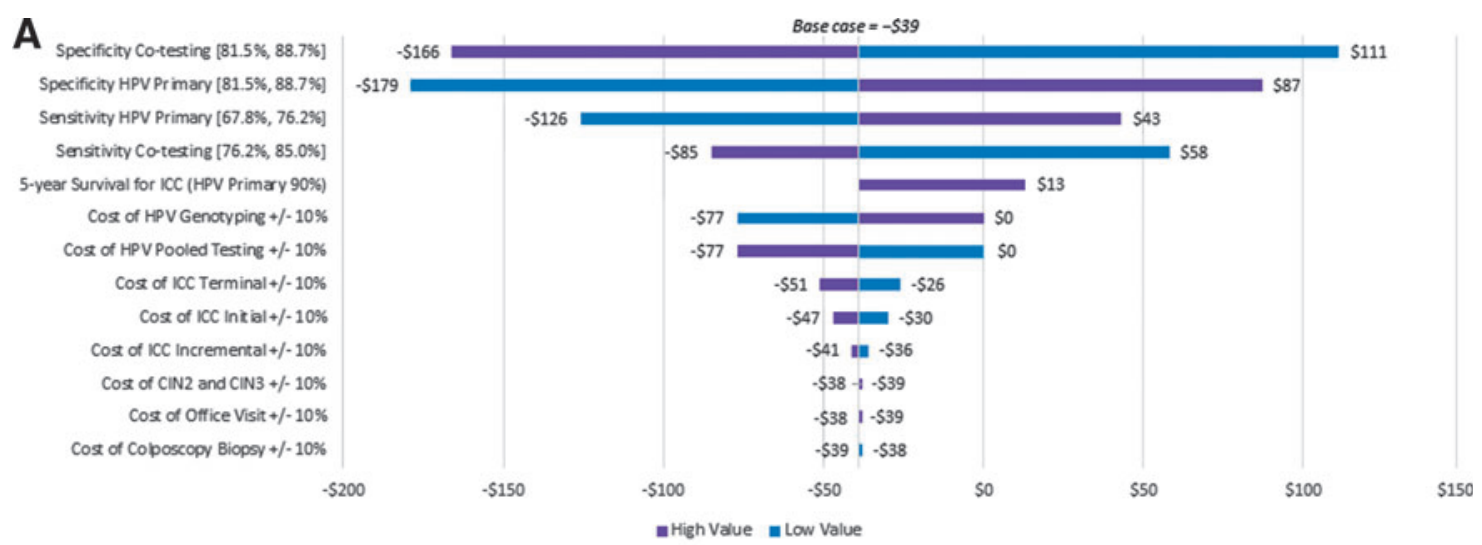

B

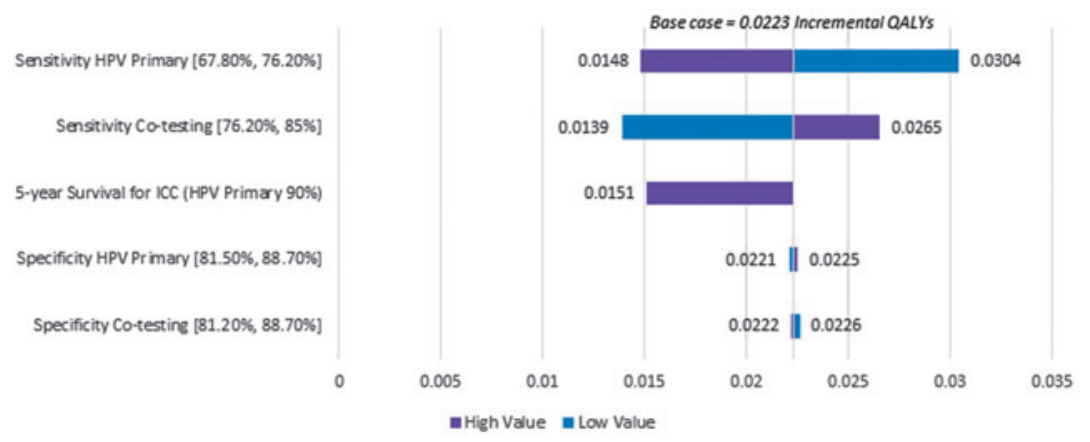

C

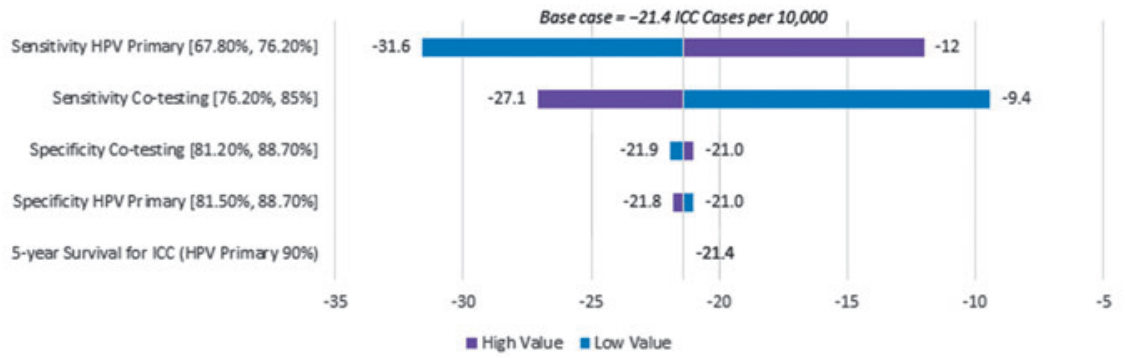

D

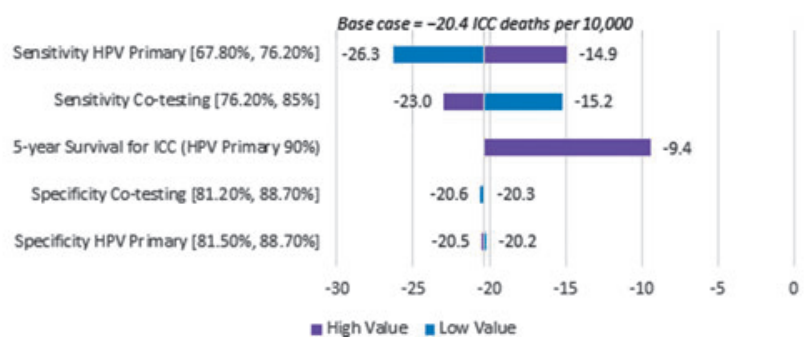

FIG. 1. One-way sensitivity analyses. (A) Sensitivity analysis of model parameter variation on net cost savings. (B) Sensitivity analysis of model parameter variation on incremental QALYs. (C) Sensitivity analysis of model parameter variation on number of ICC cases per 10,000 women. (D) Sensitivity analysis of model parameter variation on number of ICC deaths per 10,000 women.

analysis results was small (Supplementary Table S3). As expected, the number of ICC cases remained unchanged relative to the base-case analysis, but the number of ICC deaths decreased by about 33\% and 30\% for co-testing and HPV primary respectively. The impact on QALYs was negligible and the impact on costs was small, with the net difference in total costs only decreasing from $\$ 39$ in cost savings per woman to $\$ 15$ in cost savings per woman over the 40 -year simulation time horizon. In terms of cost effectiveness, co-testing con- ferred greater benefit (more QALYs) at less cost compared with HPV primary and therefore remained the economically dominant screening strategy.

Although useful for benchmarking purposes, as described here, notable limitations of the SEER mortality data include the fact that the data are 15-28 years old and geographically limited (collected in 1988 to 2001 from 12 SEER geographic areas covering only about $14 \%$ of the total U.S. population), the data pertain specifically to women diagnosed with 
squamous carcinoma of the cervix uteri, and the data were derived from the pool of women who may or may not have been screened by co-testing, HPV primary, or another screening method prior to their diagnosis.

\section{Discussion}

As discussions about appropriate cervical cancer screening strategies examine net outcomes by balancing benefits and harms, clinical-economic comparisons are useful to guide decision making. This model demonstrates that among women 30 to 70 years of age, cervical cancer screening using co-testing at 3-year intervals may be more cost effective and prevent more cases of cervical cancer compared with primary HPV screening at 3-year intervals. Co-testing dominated the HPV primary screening strategy, with lower lifetime screening and management costs and greater effectiveness as determined by ICC cases and deaths without increasing harms as measured by lifetime colposcopies/woman.

The model predicted an additional 2,141 ICC cases and 2,041 ICC deaths over 40 years with HPV primary compared with co-testing among the hypothetical one-million-woman cohort. These results are due to the greater sensitivity of cotesting compared with HPV primary. The small difference between ICC cases and deaths is the result of an increased likelihood of detecting cancer at a later, less treatable stage using HPV primary. Projected nationally, these results predict a substantial decrease in human and monetary costs over a lifetime of screening. Additionally, slightly higher QALYs per woman were calculated for co-testing. Sensitivity analyses determined that test sensitivity for both HPV primary and cotesting had a greater impact than specificity or ICC survival rate in determining QALYs or ICC mortality. These results suggest that test sensitivity is vital to consider when attempting to improve outcomes of cervical screening programs.

Calculations for cost effectiveness generated similar results for HPV primary testing and co-testing, with an approximate savings of $\$ 39$ per woman, over 40 years, using cotesting. Improvements in total costs were most sensitive to variations in test specificity followed by test sensitivity. With cost differences approaching neutral (i.e., \$39 cost savings in the base case, and a tight range of $-\$ 179$ to $\$ 111$ per screened woman in the sensitivity analyses), comparisons should focus on the improved clinical outcomes and net health benefits of co-testing. In the model, co-testing provided superior clinical outcomes (greater QALYs, fewer ICC cases, fewer ICC deaths) compared with HPV primary across a range of test performance characteristics.

Regarding strategies for women between 25 and 29 years of age, a sensitivity analysis evaluating cost effectiveness of cytology with ASCUS reflex and HPV primary determined that HPV primary was not cost effective compared with cytology with ASCUS reflex in this age group. A DNA HPV test was approved for use as a primary screen in women $\geq 25$ years of age, ${ }^{9}$ even though HPV testing of women under 30 years of age is controversial due to the additional harms and lack of established additional clinical benefit associated with HPV testing. 7,39 These data provide further evidence that while HPV primary testing in women 25 to 29 years of age is likely to identify more cases of CIN2/3, very few of these CIN2/3 cases will progress to ICC before the age of 30 . Thus, the model shows that HPV primary testing in this age group offers minimal benefit in cancer detection, does not decrease mortality from cervical cancer, and is not cost effective compared with cytological testing. Additionally, HPV primary testing in this age group resulted in a higher number of colposcopies. While the harms of excess colposcopies were not modeled here, recommendations that women younger than 30 years of age not be tested for HPV are the result of data suggesting that the harms associated with overtreatment are a significant consideration in this population. ${ }^{7}$

This analysis has several advantages over prior clinicaleconomic models that have evaluated HPV primary screening as an alternative cervical screening strategy for women in the United States. An analysis published by Huh et al. in 2015 using data from the ATHENA trial compared cost effectiveness of HPV primary testing with genotyping and cytology triage to HPV primary testing with cytology triage only and cotesting without genotyping in a hypothetical cohort of women, leading to a conclusion that use of an HPV test with genotyping improves cost effectiveness. ${ }^{15,40}$ However, direct comparison between HPV primary testing vs co-testing using HPV tests with genotyping for both strategies was not assessed until this study. A cost-effectiveness study conducted using data from a UK study found that HPV primary screening may be cost effective compared with cytology alone, but the study was performed in a population with a national health system, with markedly differing cervical screening compliance compared with the United States. ${ }^{12}$ A cost-effectiveness analysis using a Dutch model found that HPV screening was cost effective compared with cytology alone, but the authors cautioned that HPV primary screening is affected by being "well controlled" and evenly distributed throughout the population. ${ }^{41}$ Thus, caution should be used when extrapolating from European to U.S. cost-effectiveness studies.

There are several limitations to this model. As mentioned above, unscreened and underscreened women were not considered. This is relevant, as approximately half of all cervical cancer cases in the United States occur in this population. ${ }^{42}$ Assumptions of $100 \%$ sensitivity and specificity for colposcopy and $100 \%$ success for CIN2/3 treatment also limit the application of model results to real-world conditions, as does our failure to account for the impact of increasing rates of HPV vaccination. ${ }^{43} \mathrm{HPV}$ vaccination rates will affect the cervical cancer rate as well as the cost effectiveness of cervical cancer screening strategies. Finally, this analysis is limited by the lack of long-term data regarding HPV primary screening. Large-scale longitudinal studies are needed to determine the effectiveness of HPV primary screening strategies in U.S. women.

In summary, mRNA-based co-testing with HPV 16/18 genotyping dominated the DNA-based HPV primary screening with HPV 16/18 genotyping, with lower lifetime screening and management costs combined with fewer colposcopies as well as lower ICC incidence and mortality. Although it identifies more cases of CIN2/3, the addition of HPV testing to cytology in women 25 to 29 years of age provides minimal benefit in cancer detection, does not decrease mortality from cervical cancer, and is not cost effective compared with cytological testing.

\section{Conclusion}

More than 50 years after the introduction of the Pap test, cervical cancer remains a significant public health burden, 
underscoring the need for a clinically effective screening approach. The recent introduction of an HPV test for primary screening has re-opened the discussion regarding the optimal cervical screening paradigm. The results of this model demonstrate that co-testing has the potential to provide improved clinical and economic outcomes when compared with HPV primary. While actual cost and outcome data are evaluated, these findings are relevant to U.S. healthcare payers and women's health policy advocates seeking cost-effective cervical cancer screening technologies.

\section{Acknowledgment}

The authors gratefully acknowledge the contribution of Scott Hayworth, who reviewed and provided valuable comments, guidance, and direction in the development of this article.

\section{Author Disclosure Statement}

JCF and MS have received payment as consultants for Hologic, Inc. (Marlborough, MA), but received no compensation for their contributions to this study. MJL, JDM, GML, and RK were employed at the time the study was conducted by Truven Health Analytics, which received research funding from Hologic, Inc.

\section{References}

1. Siegel RL, Miller KD, Jemal A. Cancer statistics, 2015. CA Cancer J Clin 2015;65:5-29.

2. American Cancer Society. Cancer Facts and Figures 2015. Available at: www.cancer.org/research/cancerfactsstatistics/ cancerfactsfigures2015/index. Accessed January 8, 2015.

3. Riethmuller D, Jacquard AC, Lacau St Guily J, et al. Potential impact of a nonavalent HPV vaccine on the occurrence of HPV-related diseases in France. BMC Public Health 2015; $15: 453$.

4. Dunne EF, Naleway A, Smith N, et al. Reduction in human papillomavirus vaccine type prevalence among young women screened for cervical cancer in an integrated US healthcare delivery system in 2007 and 2012-2013. J Infect Dis 2015. [Epub ahead of print].

5. Gage JC, Schiffman M, Katki HA, et al. Reassurance against future risk of precancer and cancer conferred by a negative human papillomavirus test. J Nat Cancer Inst 2014; 106: dju153.

6. Blatt AJ, Kennedy R, Luff RD, Austin RM, Rabin DS. Comparison of cervical cancer screening results among 256,648 women in multiple clinical practices. Cancer Cytopathol 2015;123:282-288.

7. Saslow D, Solomon D, Lawson HW, et al. American Cancer Society, American Society for Colposcopy and Cervical Pathology, and American Society for Clinical Pathology screening guidelines for the prevention and early detection of cervical cancer. Am J Clin Pathol 2012;137: 516-542.

8. Kinney W, Wright TC, Dinkelspiel HE, DeFrancesco M, Thomas Cox J, Huh W. Increased cervical cancer risk associated with screening at longer intervals. Obstet Gynecol 2015;125:311-315.

9. U.S. Food and Drug Administration (FDA). FDA approves first human papillomavirus test for primary cervical cancer screening. FDA News Release 2014. Available at: www
.fda.gov/NewsEvents/Newsroom/PressAnnouncements/ ucm394773.htm. Accessed June 10, 2015.

10. Ronco G, Dillner J, Elfstrom KM, et al. Efficacy of HPVbased screening for prevention of invasive cervical cancer: Follow-up of four European randomised controlled trials. Lancet 2014;383:524-532.

11. Kitchener HC, Gilham C, Sargent A, et al. A comparison of HPV DNA testing and liquid based cytology over three rounds of primary cervical screening: Extended follow up in the ARTISTIC trial. Eur J Cancer 2011;47:864-871.

12. Kitchener HC, Canfell K, Gilham C, et al. The clinical effectiveness and cost-effectiveness of primary human papillomavirus cervical screening in England: Extended follow-up of the ARTISTIC randomised trial cohort through three screening rounds. Health Technol Assess 2014;18:1-196.

13. Health and Social Care Information Centre (HSCIC). Cervical screening coverage: Lowest levels amongst women under 30. HSCIC News Archive 2014. Available at: www .hscic.gov.uk/article/5215/Cervical-screening-coveragelowest-levels-amongst-women-under-30. Accessed June $10,2015$.

14. Millions of U.S. women are not getting screened for cervical cancer. CDC Newsroom Releases 2014. Available at: www.cdc.gov/media/releases/2014/p1105-vs-cervicalcancer.html. Accessed June 10, 2015.

15. Huh WK, Williams E, Huang J, Bramley T, Poulios N. Cost effectiveness of human papillomavirus-16/18 genotyping in cervical cancer screening. Appl Health Econ Health Policy 2015;13:95-107.

16. College of American Pathologists (CAP). Human papillomavirus for cytology laboratories CHPVD, CHPVM, CHPVK, CHPVJ Participant Summary. Washington, DC: CAP, 2015.

17. Kulasingam SL, Havrilesky LJ, Ghebre R, Myers ER. Screening for cervical cancer: A modeling study for the US Preventive Services Task Force. J Lower Genit Tract Dis 2013;17:193-202.

18. National Association of Nurse Practitioners in Women's Health (NPWH). HCP Cervical Cancer Survey. Washington, DC: NPWH, 2015.

19. Verrilli L, Winer RL, Mao C. Adherence to cervical cancer screening guidelines by gynecologists in the Pacific Northwest. J Low Genit Tract Dis 2014;18:228-234.

20. King NR, Kasper KM, Daggy JK, Tucker Edmonds B. Current practice patterns in cervical cancer screening in Indiana. Am J Obstet Gynecol 2014;210:265.e1-8.

21. Huh WK, Ault KA, Chelmow D, et al. Use of primary highrisk human papillomavirus testing for cervical cancer screening: Interim clinical guidance. Gynecol Oncol 2015; 136:178-182.

22. Walboomers JM, Jacobs MV, Manos MM, et al. Human papillomavirus is a necessary cause of invasive cervical cancer worldwide. J Pathol 1999;189:12-19.

23. Kulasingam SL, Havrilesky L, Ghebre R, Myers ER. Screening for cervical cancer: A decision analysis for the U.S. Preventive Services Task Force. Evidence Syntheses 86s. Rockville, MD: Agency for Healthcare Research and Quality, 2011.

24. Centers for Disease Control and Prevention (CDC). Vital signs: Cervical cancer is preventable. Atlanta: CDC, 2014. Available at: www.cdc.gov/vitalsigns/pdf/2014-11-vitalsigns .pdf. Accessed March 9, 2016.

25. Documet P, Bear TM, Flatt JD, Macia L, Trauth J, Ricci EM. The association of social support and education with 
breast and cervical cancer screening. Health Educ Behav 2015;42:55-64.

26. Campos NG, Burger EA, Sy S, et al. An updated natural history model of cervical cancer: Derivation of model parameters. Am J Epidemiol 2014;180:545-555.

27. Myers ER, McCrory DC, Nanda K, Bastian L, Matchar DB. Mathematical model for the natural history of human papillomavirus infection and cervical carcinogenesis. Am J Epidemiol 2000;151:1158-1171.

28. Cox JT, Castle PE, Behrens CM, et al. Comparison of cervical cancer screening strategies incorporating different combinations of cytology, HPV testing, and genotyping for HPV 16/18: Results from the ATHENA HPV study. Am J Obstet Gynecol 2013;208:184.e1-184.e11.

29. Hologic Inc. ThinPrep ${ }^{\circledR}$ Imaging System summary of safety and effectiveness data. Premarket approval application (PMA) number P020002. June 6, 2003.

30. Ting J, Smith JS, Myers ER. Cost-effectiveness of high-risk human papillomavirus testing with messenger RNA versus DNA under United States Guidelines for Cervical Cancer Screening. J Low Genit Tract Dis 2015;19:333-339.

31. Monsonego J, Hudgens MG, Zerat L, et al. Evaluation of oncogenic human papillomavirus RNA and DNA tests with liquid-based cytology in primary cervical cancer screening: The FASE study. Int J Cancer 2011;129:691-701.

32. Iftner T, Becker S, Neis KJ, et al. Head-to-head comparison of the RNA-based Aptima Human Papillomavirus (HPV) Assay and the DNA-based Hybrid Capture 2 HPV Test in a routine screening population of women Aged 30 to 60 years in Germany. J Clin Microbiol 2015;53:2509-2516.

33. Hologic Inc. Aptima ${ }^{\circledR}$ HPV Assay package insert \#503789 Rev A. Marlborough, MA: Hologic, 2013.

34. Vijayaraghavan A, Efrusy MB, Goodman KA, Santas CC, Huh WK. Cost-effectiveness of using human papillomavirus 16/18 genotype triage in cervical cancer screening. Gynecol Oncol 2010;119:237-242.

35. Mariotto AB, Yabroff KR, Shao Y, Feuer EJ, Brown ML. Projections of the cost of cancer care in the United States: 2010-2020. J Natl Cancer Inst 2011;103:117-128.

36. Simoens S. How to assess the value of medicines? Front Pharmacol 2010;1:115.

37. Howden LM, Meyer JA. Age and sex composition: 2010. Census Briefs 2010. Washington, DC: U.S. Department of Commerce, U.S. Census Bureau, 2011. Available at http:// www.census.gov/prod/cen2010/briefs/c2010br-03.pdf Accessed March 21, 2016.

38. Kosary CL. Cancer of the cervix uteri. In: LAG R, ed. SEER survival monograph: Cancer survival among adults: US SEER Program 1988-2001, patient and tumor characteristics. Bethesda, MD: National Cancer Institute SEER Program, 2007:111-122.

39. Sawaya GF, Kuppermann M. Identifying a "range of reasonable options" for cervical cancer screening. Obstet Gynecol 2015;125:308-310.

40. Schiffman M, Boyle S, Raine-Bennett T, et al. The role of human papillomavirus genotyping in cervical cancer screening: A large-scale evaluation of the cobas HPV test. Cancer Epidemiol Biomarkers Prev 2015;24:1304-1310.

41. de Kok IM, van Rosmalen J, Dillner J, et al. Primary screening for human papillomavirus compared with cytology screening for cervical cancer in European settings: Cost effectiveness analysis based on a Dutch microsimulation model. BMJ 2012;344:e670.

42. Leyden WA, Manos MM, Geiger AM, et al. Cervical cancer in women with comprehensive health care access: attributable factors in the screening process. J Natl Cancer Inst 2005;97:675-683.

43. Stokley S, Jeyarajah J, Yankey D, et al. Human papillomavirus vaccination coverage among adolescents, 2007-2013, and postlicensure vaccine safety monitoring, 2006-2014-United States. MMWR Morb Mortal Wkly Rep 2014;63:620-624.

44. US Social Security Administration. Actuarial Life Tables: Table 4.C6-Period life table, 2010. Available at: https:// www.ssa.gov/policy/docs/.../4c.xlsx. Accessed June 10, 2015.

45. Howlander N, Noone AM, Krapcho M, et al. SEER Cancer Statistics Review, 1975-2012, National Cancer Institute. Bethesda, MD. Available at: http://seer.cancer.gov/csr/1975_ 2012/. Accessed June 10, 2015.

Address correspondence to: Jeffrey D. Miller, MS Truven Health Analytics 150 Cambridge Park Drive Cambridge, MA 02140

E-mail: jeffrey.d.miller@truvenhealth.com 